\title{
Acute haemodynamic effects of nifedipine at rest and during maximal exercise in patients with chronic cor pulmonale
}

\author{
H SINGH, MJEBEJER, DA HIGGINS, AH HENDERSON, IA CAMPBELL \\ From the Department of Cardiology, University of Wales College of Medicine, and the Thoracic Unit, \\ Llandough Hospital, Cardiff
}

ABSTRACT The pulmonary hypertension of cor pulmonale can be reversed by sustained correction of hypoxia but continuous oxygen treatment poses problems in clinical practice. Alternative methods of relieving pulmonary vasoconstriction have therefore been explored. Eight patients with chronic cor pulmonale (five of them men) were studied to measure the haemodynamic effects of the calcium antagonist nifedipine, both at rest and on maximal, symptom limited exercise. The mean duration of exercise was unchanged by nifedipine (7.8 (SD 3.3) compared with 7.3 (3.1) min). Cardiac output rose from $5.2(1.5) 1 \mathrm{~min}^{-1}$ to $8.6(3.3) 1 \mathrm{~min}^{-1}$ on exercise. Nifedipine increased resting cardiac output by $26 \%$, but did not influence maximal exercise output. It did not significantly alter resting mean pulmonary artery pressure but reduced the level during exercise from $67(15)$ to 52(11) $\mathrm{mm} \mathrm{Hg}$. Nifedipine lowered resting pulmonary vascular resistance (PVR) by $32 \%$ and exercise PVR by $28 \%$. It reduced supine mean systemic arterial pressure by $17 \%$, standing pressure by $22 \%$, and pressure at the maximal exercise level by $20 \%$. Nifedipine lowered supine systemic vascular resistance (SVR) by $35 \%$, standing SVR by $28 \%$, and exercise SVR by $20 \%$. Haemodynamic changes were achieved without adverse symptoms, alteration in arterial $\mathbf{P o}_{2}$, or impairment of calculated oxygen delivery. Nifedipine therefore reduced both pulmonary and systemic vasomotor tone at rest and during exercise. It did not alter exercise tolerance, which is probably limited by underlying respiratory disease. It seems possible therefore that nifedipine could delay the development of cor pulmonale, although this hypothesis remains to be tested.

Hypoxia from chronic lung disease leads to pulmonary vasoconstriction, pulmonary arterial hypertension, and ultimately cor pulmonale. Animal ${ }^{1}$ and human $^{2}$ studies suggest that the pulmonary hypertension can be reversed by sustained correction of hypoxia, though the continuous oxygen treatment that appears to be necessary ${ }^{3}$ poses problems in clinical practice. Alternative methods of relieving hypoxic pulmonary vasoconstriction have therefore been explored. Rubin and Peter ${ }^{4}$ showed that hydralazine reduced pulmonary vascular resistance in patients with chronic obstructive lung disease studied at rest and during exercise. Brent et $a l^{5}$ reported a fall in pulmonary vascular resistance and improvement in

Address for reprint requests: Dr H Singh, Department of Cardiology, University of Wales College of Medicine, Cardiff CF44XN.

Accepted I July 1985 the right ventricular ejection fraction at rest with terbutaline, a $\beta_{2}$ adrenergic agonist. The calcium antagonist verapamil induced a fall in resting pulmonary vascular resistance and cardiac output in hypoxic patients. ${ }^{6}$ Nifedipine likewise reduced pulmonary vasoconstriction in patients with acute respiratory failure, ${ }^{7}$ and in patients with chronic cor pulmonale, $\widetilde{N}$ where cardiac output also rose, and the effect was $N$ maintained after nearly two months of treatment; ${ }^{8}$ 今 mean pulmonary artery pressure fell in less than half of the patients studied, however, and some patients deteriorated clinically, for reasons which were not evi- $\stackrel{\varrho}{\mathcal{D}}$ dent from the haemodynamic effects of nifedipine, $\stackrel{\mathbb{P}}{+}$ which had been measured only at rest. We therefore undertook this study of patients with chronic cor 0 pulmonale to measure the haemodynamic effects of $\overrightarrow{\mathbb{D}}$ nifedipine both at rest and at the maximal exercise $\frac{\Omega}{\mathbb{D}}$ level. Nifedipine has since been reported to reduce 
pulmonary vasoconstriction at rest and during submaximal exercise in patients with chronic obstructive lung disease. ${ }^{9}$

\section{Methods}

Eight patients (five of them men; 42-70 years) with chronic cor pulmonale were studied after giving informed written consent. The study had been approved by the hospital ethical committee. All patients had chronic airflow limitation, three also had bronchiectasis, and all had experienced at least one documented episode of clinical cor pulmonale characterised by raised venous pressure and peripheral oedema. At the time of the study all patients were in a stable state and had had no acute exacerbation within the previous three weeks. No patient had overt oedema. Treatment consisted of maintenance diuretic treatment in seven of the eight patients (frusemide 40-200 mg daily, with additional spironolactone in two patients), at least one bronchodilator in all patients, domiciliary intermittent oxygen treatment in six, digoxin in two, and maintenance oral prednisolone in one patient. Mean (SE) baseline values for

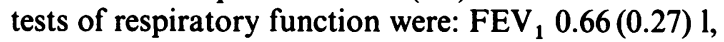
forced vital capacity (FVC) 1.43(0.66) 1 , arterial oxygen tension $\left(\mathrm{PaO}_{2}\right) 47(6) \mathrm{mm} \mathrm{Hg},(6.3(0.8) \mathrm{kPa})$, arterial carbon dioxide tension $\left(\mathrm{PaCO}_{2}\right) 53(7) \mathrm{mm} \mathrm{Hg}$ $(7.1(0.8) \mathrm{kPa})$. Radiographically, two patients showed cardiac enlargement and six showed evidence of pulmonary hypertension (diameter of one or both lower lobe pulmonary arteries $>18 \mathrm{~mm}$ ). Electrocardiographic features of pulmonary hypertension $-p$ pulmonale and/or right axis deviation $\left(>100^{\circ}\right)$ and/or right ventricular hypertrophy voltage criteria-were present in three patients.

Patients were admitted to the investigation room and rested supine until the study (for about three hours). They were studied while breathing room air, diuretics having been withheld for 24 hours and other treatment (including oxygen) for 12 hours. In each patient a Swan Ganz triple lumen thermodilution catheter was advanced from an antecubital vein to the pulmonary artery and a radial artery cannula was inserted.

Vascular pressures, with the mid chest as reference point, were measured with Gould P50 transducers connected to a Physio-control VSM 1 monitor; measurements were averaged over five respiratory cycles. Mean systemic arterial pressures were obtained electronically; because of considerable respiratory swings in pulmonary arterial pressure, means were calculated manually from phasic pressures in mid expiration. Cardiac output was measured from thermodilution data. Values that varied by more than $10 \%$ were discarded and an average was obtained from at least three acceptable measurements. Left atrial pressure was not measured (and is here ignored as it is unreliably estimated from pulmonary wedge pressure in these patients). The derived haemodynamic indices were: Total pulmonary vascular resistance $\left(\right.$ dyn $\left.\mathrm{s} \mathrm{cm}^{-5}\right)=$ $\frac{\text { mean pulmonary artery pressure }(\mathrm{mm} \mathrm{Hg}) \times 80}{\text { cardiac output }\left(1 \mathrm{~min}^{-1}\right)}$

Systemic vascular resistance $\left(\right.$ dyn s $\left.\mathrm{cm}^{-5}\right)=$ $\frac{\text { mean radial artery pressure }(\mathrm{mm} \mathrm{Hg}) \times 80}{\text { cardiac output }\left(1 \mathrm{~min}^{-1}\right)}$

Oxygen delivery $\left(\mathrm{ml} \mathrm{min}^{-1}\right)=$ cardiac output $\times$ $\left[\left(1.34 \times \mathrm{Hb} \times\right.\right.$ arterial $\mathrm{O}_{2}$ saturation \% $)+(0.003 \times$ $\left.\left.\mathrm{PaO}_{2}\right)\right]$,

where cardiac output is measured in $1 \mathrm{~min}^{-1}$, haemoglobin $(\mathrm{Hb})$ in $\mathrm{g} \mathrm{dl}^{-1}$, and $\mathrm{PaO}_{2}$ in $\mathrm{mm} \mathrm{Hg}$.

Arterial and mixed venous blood gas tensions were measured with Radiometer electrodes. Oxygen saturation was calculated from temperature corrected blood gas data on the basis of Hill's equation. ${ }^{10}$

Exercise tolerance was individually assessed by two practice tests on the day before the study. These practice tests also served to minimise the learning or training effect of repeated testing on exercise tolerance. A training effect has been found in healthy subjects, ${ }^{11}$ but not always in patients with limited exercise tolerance. ${ }^{12} \mathrm{~A}$ work load was fixed to allow each patient to exercise for at least three minutes. The exercise protocol before and after nifedipine was identical, with the patient encouraged to pedal for as long as he was able. Exercise haemodynamic measurements were made in the last 90 seconds of exercise.

Resting supine haemodynamic measurements were made at 15 minute intervals until stable recordings were obtained (after 15 minutes in six patients, after 30 minutes in two). The study was then performed according to the protocol shown in figure 1. Haemodynamic measurements were made and blood was sampled for gas analysis, in the supine position, after the patient had stood for three minutes, and at the maximal symptom limited exercise level on the bicycle ergometer. The patient then rested supine until haemodynamic measurements returned to baseline after some $20-30$ minutes. Nifedipine $(20 \mathrm{mg})$ was then given sublingually. One hour later the patients had the measurements repeated in the supine and standing positions and during exercise.

Results are given as means with standard errors in parentheses and compared with Student's $t$ test for paired data. The validity of the $t$ test results was confirmed with two way analysis of variance and Tukey's procedure for multiple comparisons.

\section{Results}

The mean duration of symptom limited exercise was 


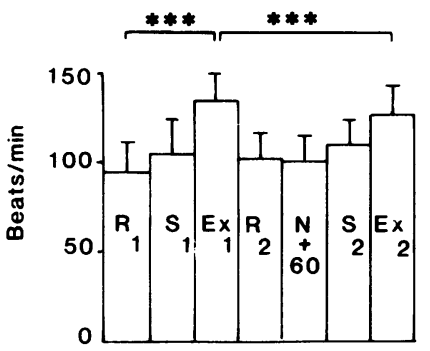

(a) Heart rate

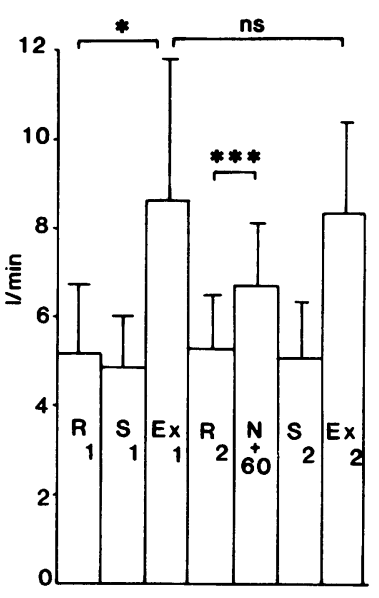

(b) Cardiac output
Fig 1 Data on heart rate and cardiac output at rest supine, standing, and during exercise before and after administration of nifedipine in eight patients with chronic cor pulmonale. Stages at which haemodynamic measurements were made: $R_{1}$-baseline, supine; $S_{1}$-after standing for three minutes; $E X_{1}$-during maximal exercise; $R_{2}$-repeat baseline, supine, after about 20 minutes; $N+60$-supine, 60 minutes after sublingual nifedipine $20 \mathrm{mg}$; $\mathrm{S}_{2}$-after standing for three minutes immediately after $N+60$ recordings; $E X_{2}-$ at maximal exercise, started immediately after $S_{2}$. Statistical comparisons between states indicated by horizontal bars: ${ }^{*} p<0.05 ;{ }^{* *} p<0.01$; ${ }^{* * *} p<0.005 ;{ }^{* * * *} p<0.001$. unchanged by nifedipine (7.8 (3.3) min compared with 7.3(3.1) min). The predominant symptom that limited exercise before nifedipine treatment was breathlessness in six patients and fatigue in two. After nifedipine, breathlessness was the limiting symptom in four and fatigue in four patients.

The heart rate increased from 95(17) beats/min while patients were resting supine to $136(15)$ during maximum exercise (fig $1 a$ ). Nifedipine did not alter the resting heart rate but lowered the exercise heart rate. Cardiac output rose from a normal level of 5.2(1.5)

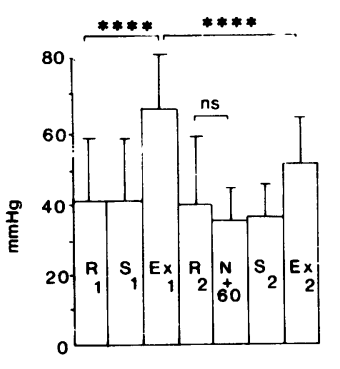

(a) Mean pulmonary artery pressure

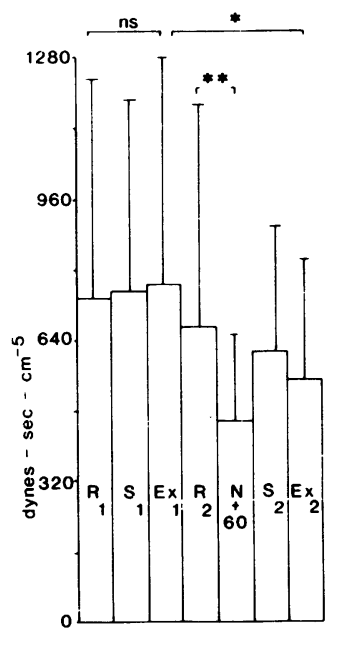

(b) PVR
Fig 2 Pulmonary artery pressure and vascular resistance. Abbreviations and conditions as in figure 1.
$1 \min ^{-1}$ while they were resting supine to $8.6(3.3)$ $1 \mathrm{~min}^{-1}$ during exercise (fig $1 \mathrm{~b}$ ). Nifedipine increased resting cardiac output by $26 \%$, but did not influence maximal exercise output. Readings for cardiac output during exercise did not show any greater variability than measurements made during rest.

Mean pulmonary pressure was high at rest (41 (17) $\mathrm{mm} \mathrm{Hg}$ ) and rose with exercise to $67(15) \mathrm{mm} \mathrm{Hg}$ (fig $2 a$ ). Nifedipine did not alter resting pulmonary pressure significantly but reduced the exercise level to 52 (11) $\mathrm{mm} \mathrm{Hg}$ (a reduction of $22 \%$ ). Pulmonary vascular resistance (PVR) rose only slightly and not significantly with exercise (from 736 to 768 dyn $\mathrm{cm}^{-5}$ ) (fig $2 b$ ). Nifedipine lowered resting PVR by $32 \%$ and exercise PVR by $28 \%$.

Nifedipine reduced resting supine systemic arterial mean pressure by $17 \%$, standing pressure by $22 \%$, and pressure at the maximal exercise level by $20 \%$ (fig $3 a$ ), without causing any symptoms of postural hypotension. Systemic vascular resistance (SVR), unlike PVR, rose on standing and fell with maximal exercise. Nifedipine lowered supine SVR by $35 \%$, standing SVR by $28 \%$, and exercise SVR by $20 \%$.

Haemodynamic changes were achieved without any significant alteration in arterial oxygen tension before or after nifedipine (fig $4 a$ ). Calculated oxygen delivery was not impaired by treatment with nifedipine (fig $4 b$ ).

\section{Discussion}

Previous studies have reported salutary effects of $\stackrel{0}{\frac{0}{\varnothing}}$ nifedipine in acute respiratory failure at rest and in $\stackrel{\mathcal{D}}{+}$ clinically stable cor pulmonale at rest and during 0 submaximal exercise. Our study extends these observations by showing that haemodynamic benefits are $\mathbb{D}$ maintained at the maximal exercise level when 


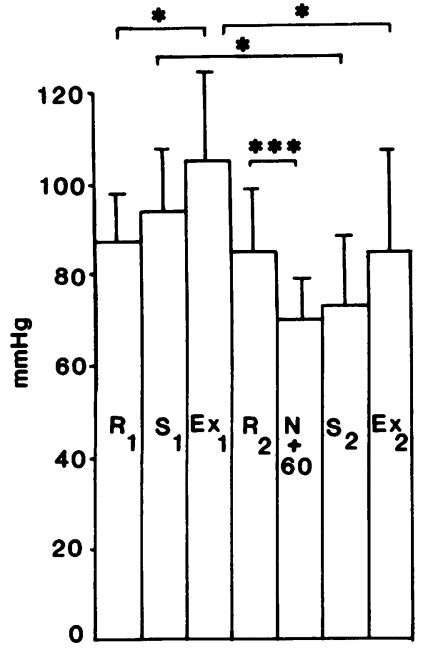

(a) Mean systemic arterial pressure

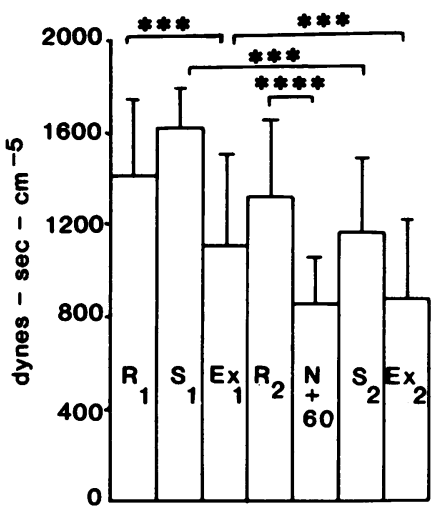

(b) SVR
Fig 3 Systemic arterial pressure and vascular resistance. Abbreviations and conditions as in figure 1. patients are breathing air.

Nifedipine reduced pulmonary and systemic vasomotor tone at rest and on maximal symptom limited exercise in patients with chronic cor pulmonale breathing air. The rise in cardiac output without intrapulmonary shunting or a rise in pulmonary artery pressure suggests that nifedipine had a pulmonary vasodilator effect. It did not alter exercise tolerance, probably because in these patients tolerance is limited by underlying respiratory disease. Patients were, however, able to reach maximal exercise at lower pulmonary pressure, heart rate and systemic pressure without any reduction of either arterial oxygen tension or calculated oxygen delivery. Nifedipine treatment is therefore unlikely to improve limiting symp- toms, but it appears not to exert any deleterious effects and has the advantage of reducing the raised pulmonary pressures that such patients experience, particularly during exercise. On theoretical grounds it might therefore delay the development of cor pulmonale, although this hypothesis remains to be tested in studies of long term treatment.

\section{References}

1 Kay JM. Effect of intermittent normoxia on chronic hypoxic pulmonary hypertension, right ventricular hypertrophy and polycythaemia in rats. Am Rev Respir Dis 1980;121:993-1001.

2 Abraham AS, Cole RB, Bishop JM. Reversal of pulmonary hypertension by prolonged oxygen adminis-

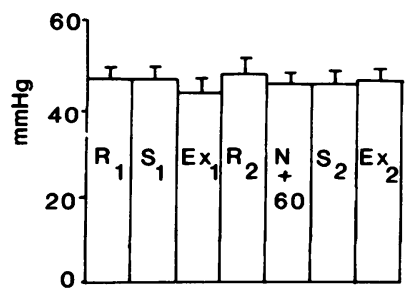

(a) Arterial $\mathrm{PO}_{2}$

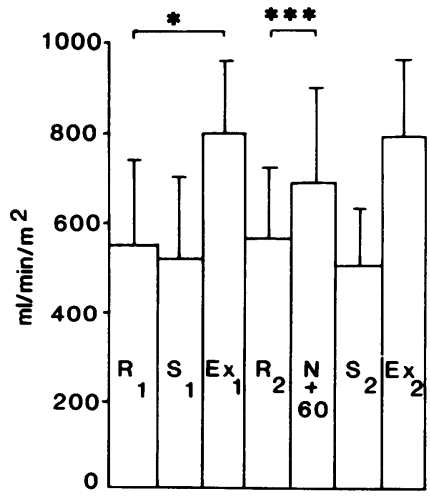

Fig 4 Arterial oxygen tension $\left(\mathrm{Po}_{2}\right)$ and calculated oxygen delivery. Abbreviations and conditions as in figure 1 .

(b) Oxygen delivery 
tration to patients with chronic bronchitis. Circ Res 1968;23:141-57.

3 Nocturnal Oxygen Therapy Trial Group. Continuous or nocturnal oxygen therapy in hypoxemic chronic obstructive lung disease. Ann Intern Med 1980;93:391-8.

4 Rubin LJ, Peter RH. Hemodynamics at rest and during exercise after oral hydralazine in patients with cor pulmonale. Am J Cardiol 1981;47:116-22.

5 Brent BN, Mahler D, Berger HJ, Matthay RA, Pytlik L, Zaret BL. Augmentation of right ventricular performance in chronic obstructive pulmonary disease by terbutaline: a combined radionuclide and hemodynamic study. Am J Cardiol 1982;50:313-9.

6 Reuben SR, Kuan P. The acute haemodynamic effects of intravenous verapamil in hypoxic lung disease (abstract). Bull Eur Physiopathol Respir 1980;16:111P-3P.

7 Simonneau G, Escourrou P, Duroux P, Lockhart A.
Inhibition of hypoxic pulmonary vasoconstriction by nifedipine. $N$ Engl J Med 1981;304:1582-5.

8 Sturani C, Bassein L, Schiavina M, Gunella G. Oral nifedipine in chronic cor pulmonale secondary to severe chronic obstructive pulmonary disease (COPD). Chest 1983;84:135-42.

9 Kennedy TP, Michael JR, Huang C-K, et al. Nifedipine inhibits hypoxic pulmonary vasoconstriction during rest and exercise in patients with chronic obstructive pulmonary disease. Am Rev Respir Dis 1984;129:544-51.

10 Berk JL, Sampliner JE, eds. Handbook of critical care. 2nd ed. Boston: Little Brown and Co, 1982:563.

11 Davies CTM, Tuxworth W, Young JM. Physiological effects of repeated exercise. Clin Sci 1970;39:247-58.

12 Dagenais GR, Pitt B, Ross RS. Exercise tolerance in patients with angina pectoris. Am $J$ Cardiol 1971;28:10-6. 